\title{
MEASURES INVARIANT UNDER LOCAL HOMEOMORPHISMS
}

\author{
DAVID ROSS ${ }^{1}$
}

(Communicated by R. Daniel Mauldin)

\begin{abstract}
Suppose $X$ is a compact Hausdorff space, and $G$ is a set of local homeomorphisms of $X$; sufficient conditions are given for the existence of a $G$ invariant Borel probability measure $P$ on $X$. The result generalizes theorems of Mycielski and Steinlage. The proof is an application of the "Loeb measure" construction from nonstandard analysis.
\end{abstract}

1. Introduction. Suppose $X$ is a compact Hausdorff space, and $G$ is a set of local homeomorphisms of $X$ : does there exist a $G$-invariant Borel probability measure $P$ on $X$ ? The answer is yes under either of the two extra hypotheses:

(i) [Mycielski] $X$ is metric, and $G$ consists of all local isometries of $X$.

(ii) [Steinlage] $G$ is a weakly transitive group of autohomeomorphisms of $X$, and for every disjoint pair $K, L$ of compact subsets of $X$ there is an open set $u$ such that for no $g \in G$ does $g u$ simultaneously intersect both $K$ and $L$.

Here, a local homeomorphism (isometry) is a homeomorphism (isometry) from one open subset of $X$ onto another, and an autohomeomorphism has range = domain $=X . P$ is $G$-invariant provided that, for each $g \in G$ and Borel subset $E$ of domain $(g), P E=P g E$. $G$ is weakly transitive provided that $X=\bigcup_{g \in G} g u$ for every open subset $u$ of $X$.

Steinlage's theorem is a strong generalization of the existence theorem for Haar measure; Mycielski's theorem is a partial solution to the second problem in the Scottish book [2]. The proofs of these results are difficult, and do not resemble one another.

This paper presents a simple proof of a theorem which simultaneously generalizes the above results. Suppose $U$ is an open cover of $X$, and $G^{\prime} \subseteq G$; call $U$. $G^{\prime}$ distributed provided whenever $u \in U, g \in G^{\prime}$, and $u \subseteq \operatorname{domain}(g), g u \in U$. If, in addition, $K^{\prime}$ is a collection of compact sets, call $U K^{\prime}$-small provided that whenever $u \in U$, and $K$ and $L$ are disjoint elements of $K^{\prime}$, then either $u \cap K=\varnothing$ or $u \cap L=\varnothing$.

THEOREM 1. Let $X$ be a compact Hausdorff space, and let $G$ be a set of local homeomorphisms of $X$, closed under inverses. Suppose that for every finite $G^{\prime} \subseteq G$, and finite collection $K^{\prime}$ of compact sets, there is a $G^{\prime}$-distributed $K^{\prime}$-small open cover $U$ of $X$. Then there is a $G$-invariant Borel probability measure $P$ on $X$.

2. Preliminaries. For simplicity, the definitions will be rephrased in the language of nonstandard analysis, and proved by means of Loeb's hyperfinite measure

Received by the editors January 13, 1987.

1980 Mathematics Subject Classification (1985 Revision). Primary 28A12, 28C10; Secondary $28 \mathrm{E} 05$

${ }^{1}$ The author is supported by an SERC grant. 
construction. The reader is referred to $[\mathbf{1}$ or $\mathbf{7}]$ for definitions, notation, and details. Some major facts are summarized here.

Suppose $X$ is compact Hausdorff and $\Omega^{*} \subseteq X$ is ${ }^{*}$ finite. If $A$ is an internal subalgebra of ${ }^{*} P(\Omega)$, the algebra of internal subsets of $\Omega$, then $\left.\overline{L(\underline{A}}\right)$ denotes the smallest (external) $\sigma$-algebra containing $\underline{\underline{A}}$. Write $L(\Omega)=L(\underline{\underline{A}})$ when $\underline{\underline{A}}={ }^{*} P(\Omega)$.

If $\mu$ is an internal *probability measure on $(\Omega, \underline{\underline{A}})$, then there is a standard probability space $(\Omega, L(\underline{\underline{A}}), L(\mu))$ such that $L(\mu)(E) \equiv \sup \left\{{ }^{\circ} \mu(A): A \in \underline{A}, A \subseteq E\right\}$ for $E \in L(\underline{\underline{A}})$; in particular, $L(\mu)(A)={ }^{\circ} \mu(A)$ for $A \in \underline{\underline{A}}$. Denote by $L^{\prime}(\underline{\underline{A}})$ the completion of $L(\underline{\underline{A}})$ under $L(\mu)$.

If $E$ is a Baire subset of $X$, and $\Omega$ is $S$-dense (that is, $X=\left\{{ }^{\circ} x: x \in \Omega\right\}$ ), then $\Omega \cap \mathrm{st}^{-1} E \in L(\Omega)$. Thus, if $\mu$ is an internal probability measure on $\left(\Omega,{ }^{*} P(\Omega)\right)$, then there is a natural image Baire probability measure $P$ on $X$, given by $P(E)=$ $L(\mu)\left(\right.$ st $\left.^{-1} E\right)$. The completion of this Baire measure, which includes the Borel sets $B_{X}$, is the image of $\left(\Omega, L^{\prime}(\Omega), L(\mu)\right)$.

If $u \subseteq{ }^{*} X$, then call $u$ an infinitesimal neighborhood provided $u$ is *open, and whenever $v$ is an open subset of $X$ with $u \cap^{*} v \neq \varnothing, u \subseteq{ }^{*} v$. Assuming the nonstandard model is sufficiently saturated, every $x \in X$ is contained in an infinitesimal neighborhood. If $g$ is a local homeomorphism of $X$, and $u$ is an infinitesimal neighborhood contained in ${ }^{*}$ domain $(g)$, then ${ }^{*} g(u)$ is another infinitesimal neighborhood.

For $A$ a $^{*}$ finite set, denote by $\|A\|$ the internal cardinality of $A$. If $B$ is any (standard) set, let ${ }^{\sigma} B=\left\{{ }^{*} b: b \in B\right\}$.

\section{Proof of main result.}

LEMMA 1. Suppose $X$ is a compact Hausdorff space, and $G$ is a set of local homeomorphisms of $X$, closed under inverses. The following are equivalent:

(i) For every finite $G^{\prime} \subseteq G$, and finite collection $K^{\prime}$ of compact sets, there is a $G^{\prime}$-distributed $K^{\prime}$-small open cover $U$ of $X$.

(ii) There is a ${ }^{\sigma} G$-distributed ${ }^{*}$ open ${ }^{*}$ cover $U$ of ${ }^{*} X$, with each $u \in U$ an infinitesimal neighborhood.

PrOOF. (i) $\rightarrow$ (ii). By saturation there is a ${ }^{\sigma} G$-distributed *open *cover of ${ }^{*} X$ such that, whenever $K$ and $L$ are disjoint compact subsets of $X$ and $u \in U$, either $u \cap{ }^{*} K=\varnothing$ or $u \cap{ }^{*} L=\varnothing$. It suffices to show that this last condition ensures that every $u$ in $U$ is infinitesimal. Let $x, y \in u$, and suppose ${ }^{\circ} x \neq{ }^{\circ} y$. There is an open set $v$ containing ${ }^{\circ} x$ and an open set $w$ containing ${ }^{\circ} y$, such that the closures $K$ of $v$ and $L$ of $w$ are disjoint. However, $x \in u \cap \operatorname{st}^{-1}\left({ }^{\circ} x\right) \subseteq u \cap{ }^{*} K$ and $y \in u \cap \operatorname{st}^{-1}\left({ }^{\circ} y\right) \subseteq u \cap{ }^{*} L$, a contradiction. Thus ${ }^{\circ} x={ }^{\circ} y$, so $x \approx y$ and $u$ is infinitesimal.

(ii) $\rightarrow$ (i). Fix $G^{\prime}$ and $K^{\prime}$, and let $U$ be the ${ }^{*}$ open cover from (ii). If $g \in G^{\prime}$, then $U$ is $\left\{{ }^{*} g\right\}$-distributed so (since $G^{\prime}$ is finite) $U$ is ${ }^{*} G^{\prime}$-distributed. If $K$ and $L$ are disjoint elements of $K^{\prime}$, and $u \in U$, the Hausdorff hypothesis on $X$ guarantees that either $u \cap^{*} K=\varnothing$ or $u \cap^{*} L=\varnothing$; from this and finiteness of $K^{\prime}$ it follows that $U$ is ${ }^{*} K^{\prime}$-small. By transfer there is a $G^{\prime}$-distributed $K^{\prime}$-small open cover of $X$.

ProOF OF THEOREM 1 . By Lemma 1 let $U$ be a ${ }^{\sigma} G$-distributed *open *cover of ${ }^{*} X$. Let $\left\{u_{1}, \ldots, u_{H}\right\}$ be a ${ }^{*}$ finite subcover of ${ }^{*} X$ from $U$; such a subcover exists since $U$ is internal and ${ }^{*} X$ is ${ }^{*}$ compact. Choose this subcover so that $H$ is *minimum. 
For $i \leq H$, choose $x_{i} \in U_{i}$; this can be done in such a way that $\Omega=\left\{x_{1}, \ldots, x_{H}\right\}$ is internal, and $x_{i} \neq x_{j}$ whenever $i \neq j$. There is an internal function $u:{ }^{*} X \rightarrow U$ such that $x \in u(x)$ for all $x \in{ }^{*} X$, and $u\left(x_{i}\right)=u_{i}$ for every $i \leq H$. Evidently $\Omega$ is $S$-dense.

Let $\underline{\underline{A}}={ }^{*} P(\Omega)$, and for $A \in \underline{\underline{A}}$ put $\mu(A)=\|A\| / H$. Extend the internal *probability $(\Omega, \underline{\underline{A}}, \mu)$ to the complete probability space $\left(\Omega, L^{\prime}(\Omega), L(\mu)\right)$ by means of the Loeb construction. Let $\left(X, B_{X}, P\right)$ be the image under the standard part map of this measure. It remains to show that $P$ is $G$-invariant.

Take $g \in G, E \in B_{X}$ contained in the domain of $g$, and let $A$ be any internal subset of $\Omega \cap \mathrm{st}^{-1} E$. It suffices to produce an internal subset $B$ of $\Omega \cap \mathrm{st}^{-1} g(E)$ with $\|B\| \geq\|A\|$ (since then $P(E) \leq P\left(g(E)\right.$ ); the same argument applied to $g^{-1}$ proves $P(E)=P(g(E)))$.

For $i \leq H$ let $i^{+}=\left\{j \leq H:{ }^{*} g\left(u_{i}\right) \cap u_{j} \neq \varnothing\right\}$. Put $B=\left\{x_{j}: j \in i^{+}\right.$for some $\left.x_{i} \in A\right\}$. Since each $u_{i}$ is an infinitesimal neighborhood of $x_{i}$ whenever $x_{i} \in A$ and $j \in i^{+}$we have $u_{i} \subseteq \mathrm{st}^{-1} E$ and $u_{j} \subseteq \mathrm{st}^{-1} g(E)$. Thus $B \subseteq \mathrm{st}^{-1} g(E)$.

Observe that $\left\{u_{i}: x_{i} \in(\Omega \backslash A)\right\} \cup\left\{u(x): x \in g^{-1}(B)\right\}$ is a subcover from $U$. Since $H$ was chosen to be a minimum, $\|B\| \geq\|A\|$. The theorem is proved.

REMARK. The last paragraph of the above proof actually shows that for every internal $D \subseteq\{1, \ldots, H\},\left\|\bigcup_{i \in D} i^{+}\right\| \geq\|D\|$. Since the map $i \rightarrow i^{+}$is internal, an internal application of Hall's "Marriage Theorem" produces an internal permutation $\pi$ of $\{1, \ldots, H\}$ with $\pi(i) \in i^{+}$for all $i$. It follows that there is a map $\theta$ from $G$ into the ${ }^{*}$ group $S_{H}$ of internal permutations of $\{1, \ldots, H\}$, with the property that if $g \in G$ and $\pi=\theta(g)$ then $g\left(x_{i}\right) \approx x_{\pi(i)}$ for all $i$. With care, this $\theta$ can be made an isomorphism.

\section{Applications.}

COROLlARY 1 (MYCIELSKI'S THEOREM). Suppose $X$ is compact metric, and $G$ is the set of local isometries of $X$. Let $\varepsilon$ be positive infinitesimal, and $U$ the set of all $\varepsilon$-balls in ${ }^{*} X$. If $g \in G$ has domain $v$, and $u \in U$ is a subset of ${ }^{*} v$, then ${ }^{*} g(u)$ is an $\varepsilon$-ball because ${ }^{*} g$ is an ${ }^{*}$ isometry. $U$ is therefore ${ }^{\sigma} G$-distributed, and Mycielski's theorem follows from Theorem 1 .

COROllaRY 2 (STEINlage's THEOREM). Suppose the hypotheses of Steinlage's theorem are satisfied. Fix $G^{\prime} \subseteq G$ finite, and let $K^{\prime}$ be a finite collection of compact subsets of $X$. For every pair $\gamma=\{K, L\}$ of disjoint elements of $K^{\prime}$ there $i s$, by hypothesis, an open set $u_{\gamma}$ no image of which simultaneously intersects both $K$ and $L$. By weak transitivity of $G, U_{\gamma}=\left\{g u_{\gamma}: g \in G\right\}$ covers $X$.

Let $U$ consist of all open sets of the form $\bigcap V$, where $V$ contains exactly one element from each $U_{\gamma}$. Since each $U_{\gamma}$ is a $G$-distributed open cover of $X$, and $K^{\prime}$ is finite, $U$ is a $G$-distributed open cover of $X$. Clearly $U$ is $K^{\prime}$-small. The hypotheses of Theorem 1 are satisfied, so there is a $G$-invariant Borel probability measure on $X$.

COROllaRY 3 (HAAR MEASURE). Suppose $X$ is a compact topological group (see [4] for definitions). For any $z \in X$, let $g_{z}$ be the function $x \rightarrow x z$. Then $G=\left\{g_{z}: z \in X\right\}$ is a group of homeomorphisms of $X$. Haar measure is a $G$ invariant Borel probability measure on $X$. 
Fix any infinitesimal neighborhood $u$ in ${ }^{*} X$, and let $U=\left\{g(u): g \in{ }^{*} G\right\} . U$ is clearly $a^{\sigma} G$-distributed cover of ${ }^{*} X$. The existence of Haar measure will follow from Theorem 1 once it is proved that every element of $U$ is an infinitesimal neighborhood.

Suppose that $g=g_{z} \in{ }^{*} G, z \in{ }^{*} X$. Since $g$ is a ${ }^{*}$ homeomorphism, $g(u)$ is ${ }^{*}$ open. Take any $x, y \in u$. Note that

$$
g(x)=x z=x^{\circ} z\left({ }^{\circ} z\right)^{-1} z \approx x^{\circ} z z^{-1} z=x^{\circ} z,
$$

where continuity of the function $z \rightarrow z^{-1}$ implies that $\left({ }^{\circ} z\right)^{-1} \approx z^{-1}$. Similarly, $g(y) \approx y^{\circ} z$. Since $x \approx y, g(x) \approx g(y)$, so $g(u)$ is an infinitesimal neighborhood.

For the next application, recall that a uniformity on $X$ is a collection $T=\left\{U_{\alpha}\right\}$ of open covers of $X$ such that each pair $U_{\alpha}, U_{\beta}$ has a common barycentric refinement $U_{\gamma}$. That is, for every $x \in X$ there are sets $u \in U_{\alpha}$ and $v \in U_{\beta}$ with $U_{\gamma}[x] \subseteq(u \cap v)$, where $U_{\gamma}[x]=\bigcup\left\{w \in U_{\gamma}: x \in w\right\}$. The uniformity $T$ is compatible with $X$ provided the sets $U[x]$, where $x \in X$ and $U \in T$, form a base for the topology of $X$. Call a local homeomorphism $f$ of $X T$-preserving provided $f(u) \in U$ whenever $u \in U \in T$ and $u$ is contained in the domain of $f$.

COROLlaRY 4. Suppose $X$ is compact Hausdorff, $T=\left\{U_{\alpha}\right\}$ is a uniformity compatible with $X$, and $G$ is the set of T-preserving local homeomorphisms of $X$. By saturation there is a $U \in{ }^{*} T$ which ${ }^{*}$ refines each standard $U_{\alpha}$. By definition of $G, U$ in ${ }^{*} G$-distributed, and it is easy to see that each $u \in U$ is infinitesimal, so $X$ has a $G$-invariant Borel probability measure.

In the examples discussed so far, $X$ has a local uniform structure which $G$ preserves. The last application is a space $X$ with a natural local structure not preserved by $G$.

EXAMPLE 1 . Let $(Y, d)$ and $(T, \rho)$ be metric spaces, with $T$ compact. Fix any group $H$ of autoisometries of $Y$ with the property that if $H^{\prime}$ is a finite subset of $H$, and $K$ is a compact subset of $Y$, then $\left\{h^{n}(K): h \in H^{\prime}, n \in Z\right\}$ has compact closure. (Examples include rotations around the origin of $\mathbf{R}^{2}$, and finite permutations on a discrete space $Y$.) Let $\Gamma$ be the set of components of $Y$, and for $\gamma \in \Gamma$ denote by $I_{\gamma}$ the characteristic function of $\gamma$.

Let $X$ be the space of 1-Lipschitz functions from $Y$ to $T$; that is, $f \in X$ provided $\rho(f(y), f(z)) \leq d(y, z)$ for all $y, z \in Y$. Ascoli's theorem ensures that this space is compact in the topology of uniform convergence on compact sets. Recall that a basic open set for this topology has the form $N(f, K, \varepsilon)=\left\{f^{\prime} \in X: \rho\left(f(y), f^{\prime}(y)\right)<\varepsilon\right.$ for all $y \in K\}$, where $f \in X, K$ is a compact subset of $Y$, and $\varepsilon>0$.

Let $G$ be the set of all autohomeomorphisms of $X$ of the form $g(x)=x \circ h$, where $h \in H$, together with those of the form $g(x)=\sum_{\gamma \in \Gamma} h_{\gamma} \circ x \circ I_{\gamma}$, where for $\gamma \in \Gamma$ $h_{\gamma}$ is an autoisometry of $T$.

By saturation and the definition of $H$, there is a * compact subset of $K$ of ${ }^{*} Y$ such that, for every standard compact $K^{\prime} \subseteq Y$ and every $h \in H,{ }^{*} K^{\prime} \subseteq K={ }^{*} h(K)$. Put $\varepsilon>0$ infinitesimal, and let $U=\left\{N(f, K, \varepsilon): f \in{ }^{*} X\right\}$. Since $K$ is ${ }^{\sigma} H$-invariant, $U$ is ${ }^{\sigma} G$-distributed; since $K$ contains all standard compact sets and $\varepsilon \approx 0$, each $u \in U$ is infinitesimal. It follows from Theorem 1 that there is a $G$-invariant Borel probability measure on $X$.

Could this measure have been obtained as a consequence of Corollary 1 or 2? Suppose $H$ has the additional property (satisfied, for example, by finite permutations on a discrete $Y$ ) that if $K$ is compact then $h(K) \cap K=\varnothing$ for some $h \in H$. 
Fix $t \in T$, let $\tau$ be the constant function $\tau(y)=t$, and let $f$ be any element of $X-\{\tau\}$ with the property that $f(y)=t$ for all $y$ off some compact subset $K$ of $Y$.

Suppose $u$ is any open neighborhood of $\tau$; for some $\varepsilon>0$ and compact $K^{\prime} \subseteq Y$, $N\left(\tau, K^{\prime}, \varepsilon\right) \subseteq u$. Choose $h \in H$ satisfying $h\left(K \cup K^{\prime}\right) \cap\left(K \cup K^{\prime}\right)=\varnothing$, and let $g$ be the autohomeomorphism $g(x)=x \circ h$. Since $g(\tau)=\tau, g\left(N\left(\tau, K^{\prime}, \varepsilon\right)\right)=N\left(\tau, h\left(K^{\prime}\right), \varepsilon\right)$. Thus both $\tau$ and $f$ are elements of $g(u)$.

The disjoint pair of compact sets $\{f\}$ and $\{\tau\}$ provide an immediate counterexample to the hypotheses of Steinlage's theorem. As for Mycielski's theorem, observe that $X$ is, in general, not metrizable. Moreover, in those cases where $X$ is metrizable with a metric $\eta$, then some element of $G$ takes the $\delta$-ball around $\tau$, where $\delta=\eta(\tau, f) / 2$, onto an open set containing both $\tau$ and $f$; it follows that $G$ is not a set of isometries, so the hypotheses of Mycielski's theorem fail as well.

5. Extensions. In [6], Steinlage proves his theorem not just for compact Hausdorff spaces, but indeed for any locally compact Hausdorff space $X$. Theorem 1 extends to such spaces as well, provided the hypothesis is strengthened and the conclusion weakened as follows: In the hypothesis replace the word "compact" by the word "closed"; in the conclusion, replace "Borel" by "Baire". The proof is quite the same, though technically more involved. See [5] for a discussion of the special case where $G$ is a group of autohomeomorphisms.

The author does not know whether, in the locally compact case, Theorem 1 holds with "compact" in place of "closed".

If $X$ is a metric space and $G$ is the set of local isometries, then Theorem 1 is no stronger than Mycielski's theorem. In particular, it gives no more information about the Scottish book problem mentioned in $\S 1$. That problem is to find a measure invariant under all partial isometries, not just those with open domain and range. Christoph Bandt has shown that such a measure exists provided the space $X$ is locally homogeneous, i.e., for every $x, y \in X$ there is a local isometry $g$ of $X$ with $g(x)=y$.

6. Acknowledgments. The author is grateful to Nigel Cutland for discussion and advice, and to the referee for pointing out a terminological ambiguity in the original manuscript and for describing Bandt's result mentioned above.

\section{REFERENCES}

1. N. J. Cutland, Nonstandard measure theory and its applications, Bull. London Math. Soc. 15 (1983), 529-589.

2. R. D. Mauldin (ed.), The Scottish book, Birkhäuser, Boston, Mass., 1981.

3. J. Mycielski, Remarks on invariant measures in metric spaces, Colloq. Math. 32 (1974), 105112.

4. L. Nachbin, The Haar integral, Van Nostrand, Princeton, N.J., 1965.

5. D. Ross, Measurable transformations and saturated models of analysis, Ph.D. Thesis, University of Wisconsin, 1983.

6. R. C. Steinlage, On Haar measure in locally compact $T^{2}$ spaces, Amer. J. Math. 97 (1975), 291-307.

7. K. D. Stroyan and J. M. Bayod, Foundations of infinitesimal stochastic analysis, North-Holland, Amsterdam, 1986.

Department of Pure Mathematics, University of Hull, Hull, HU6 7RX, EngLAND 